\title{
An intensive training of cardiothoracic surgical skills and simulation course increases procedural knowledge - feedback from fifteen courses
}

\author{
Krishnamoorthy $\mathrm{B}^{1,2 *}$, Critchley $\mathrm{WR}^{3}$, Shah $\mathrm{R}^{1}$, Bartely $\mathrm{T}^{5}$, Kendall $\mathrm{S}^{6}$, Lewis $\mathrm{M}^{7}$, Walker $\mathrm{A}^{4}$ and Bose $\mathrm{A}^{4}$ \\ ${ }^{1}$ Department of Cardiothoracic Surgery, University Hospital of South Manchester NHS Foundation Trust, Manchester, UK \\ ${ }^{2}$ Faculty of Health and Social Care, Edge Hill University, Ormskirk, Lancashire, UK \\ ${ }^{3}$ The Manchester Collaborative Centre for Inflammation Research, Faculty of Medical and Human Sciences, University of Manchester, UK \\ ${ }^{4}$ Department of Cardiothoracic Surgery, Blackpool Victoria hospital, Blackpool, UK \\ ${ }^{5}$ Department of Cardiothoracic Surgery, Queen Elizabeth Birmingham, UK \\ ${ }^{6}$ Department of Cardiothoracic Surgery, James Cook University Hospital, Middlesbrough, UK \\ ${ }^{7}$ Department of Cardiothoracic Surgery, Royal Sussex County Hospital, Brighton, UK
}

\begin{abstract}
Background: A lack of incorporated teaching time in the National Health Service leaves surgical trainees and surgical care practitioners lacking confidence during surgery. We implemented a simulation based surgical skills course in cardiothoracic surgery to supplement in-post training. This study aimed to understand the ability of health care professionals and to get their constructive feedback post training.

Method: All participants underwent 10 hours of intense cardiothoracic skills training. The participants included are surgical specialist registrars, core surgical trainees and surgical care practitioners. Participants completed pre and post course Likert scale questionnaires assessing their procedural knowledge and learning outcomes. Consultant and senior surgical faculty members taught at the courses in a ratio of almost 2:1 participant to teachers. A variety of cardiothoracic skills were taught using porcine and human cadaverous surgical skills models. All the skills sessions were structured and aligned with their current surgical curriculum.

Result: Participants reported low pre-course knowledge of the topics covered in the course (study mean less than 2.07 for all topics). High satisfaction was reported with the content of the course and the organisation and logistics involved. There was a high likelihood of participants recommending the course to colleagues.

Conclusion: Pre-course cardiothoracic skills of participants were limited. This clearly identifies that the teaching time spent in the operating theatre is insufficient. All participants felt that these types of cardiothoracic surgical skills courses will increase the chance of improved patient outcomes. It is important to teach trainees with adequate anatomical demonstration and hands on practice outside the theatre settings. This type of training may be useful for other surgical specialities.
\end{abstract}

Abbreviations: CT: Cardiothoracic, CT1, CT2: Core trainees, NHS: National Health Service, SCTS: Society of Cardiothoracic Surgery, ST1, ST2: Specialist trainees, VATs: Video Assisted Thorcoscopy.

\section{Introduction}

The diminished teaching time during surgical rotation or in operating theatres leave the surgical core trainees with fewer hands on experiences with cardiothoracic (CT) surgical procedures [1]. Many specialities have adapted the new model of surgical education; basic surgical skills are carried out on models and simulators rather than on patients to prepare the trainees for the operating room [2]. However, cardiothoracic surgery remains far behind in wet lab skills teaching for the surgical core trainees and surgical care practitioners. Motor skill acquisition is very important in CT surgery and lack of these skills can lead to poor outcomes [3]. Importantly, the surgical care practitioners are exposed to a very limited number of hands-on courses during and after their surgical training period. This restricts their continuous professional development and exposure to different operative skills. In contrast, the surgical core trainees are exposed to different specialities but very limited cardiothoracic skills, leaving them as non-experts which will affect the patients' outcomes.
There are no regular cardiothoracic surgery skills wet lab programmes set up for health professionals in United Kingdom. The problem of running these wet labs in each individual hospital is that this is very expensive and time consuming. The Society of Cardiothoracic Surgery has identified the lack of surgical skills as a key problem. We have set up a curriculum aligned hands-on wet lab which includes all basic cardiothoracic surgical procedures, minimally invasive conduit harvesting, full CT anatomy cadaveric dissection and computer based laparoscopic skills [4].

${ }^{*}$ Correspondence to: Krishnamoorthy B, Lead Surgical Care Practitioner, Cardiothoracic surgery, Manchester Foundation Trust, Programme Director/ Senior Lecturer at Edgehill University for MSc Surgical Practice, Honorary Research Associate at The University of Manchester, University Hospital of South Manchester NHS Foundation Trust Manchester, UK, Tel: 0044161291 2078; Fax: 0044161291 5024; E-mail: bhuvaneswari.bibleraaj@mft.nhs.uk

Key words: core trainees, surgical care practitioners, cadaveric tissues, porcine tissues, simulation

Received: February 01, 2019; Accepted: February 11, 2019; Published: February 13, 2019 
The purpose of this article is to provide an overview of the surgical skills in cardiothoracic surgery training and also to analyse the precourse understanding of participants and delegates' feedback postcourse.

\section{Method}

All the courses were conducted in the Manchester Surgical Skills Simulation unit at The University of Manchester. All the participants provided verbal consent to use their feedback for data analysis. There were a total of fifteen courses conducted with 207 participants and involving the input of 105 consultants and senior faculty members. All participants underwent 10 hours of intense cardiothoracic skills training involving open and endovascular surgical procedures using porcine and human cadaverous skills models. This cardiothoracic intensive course was open to all surgical specialist registrars (ST1 and ST2), core surgical trainees (CT1, CT2) and surgical care practitioners. The main aim of this course was to improve the cardiothoracic surgical skills and understanding of the surgical procedures in a quiet environment. The course had three educational goals: a) to understand the current cardiothoracic surgical procedural knowledge of the trainees prior to the course, b) to understand the overall course satisfaction and also participant's satisfaction of each surgical procedure, c) to understand self-rated confidence that their patient outcomes will improve as a result of this course.

\section{Structure of the course}

This course consists of one day of hands-on intensive wet lab sessions consists of 12 various surgical procedures in cardiothoracic surgery. Five to ten minutes of lectures or diagrammatic flip chart based interactive teaching was used to debrief the plan of the sessions. To improve the experience of each participant, some of the sessions were designed for two participants to work together with a consultant. This allowed the participants to gain experience as a first assistant and also as an operating surgeon and vice versa.

\section{Cadaveric anatomy}

The participants were taught detailed cardiothoracic cadaveric human anatomy in small groups by a Professor of anatomy from The University of Manchester. They also received hands-on experience on cadaveric dissections and completed a quick test at the end of each group sessions to assess their knowledge. The cadaveric dissections were organised as heart and great vessels, thoracic cavity and lungs, mediastinal cavity and associated vascular and muscular structures. In addition to the above-mentioned dissections, the participants were also taught radial and great saphenous vein anatomy.

\section{Bronchoscopy}

The participants were taught and practised rigid and flexible bronchoscope techniques one by one with a mannequin. We have used erudite new technology HOPKINS ${ }^{\oplus}$ telescopes, IMAGE 1 S 3D camera platform and basic set of bronchoscopies which was supplied courtesy of Karl Storz ${ }^{\oplus}, \mathrm{GmbH}$, Deutschland. The participants also utilised flexible, rigid scopes and practised stent insertion under supervision of a consultant thoracic surgeon. They also practised foreign body removal, which was a really difficult task to perform on mannequins.

\section{Chest drain insertion}

The porcine full chest was used for this task and the participants were taught full anatomical drain insertion site for pleural effusion, surgical drain insertion, seldinger techniques and pneumothorax drain insertion techniques. For chest drain insertion, they used the rocket chest insertion pack and underwater seal drainage system (supplied by the courtesy of Rocket ${ }^{\oplus}$ Medical plc. Herts, England). In addition to the insertion, they were also asked to remove the chest drains and close the insertion site.

\section{Open thoracotomy and lung wedge resection}

Full porcine samples were used for the different types of thoracotomy incisions and the same samples were used for lung wedge resections and closure of the thoracotomy wounds. The participants worked in pairs and helped each other under supervision of the consultant thoracic surgeon. They have been cross-examined about the surgical skills and also thoracic surgical procedures during each session by the faculty.

\section{Endoscopic vein and radial artery harvesting}

The participants were paired up and carried out endoscopic vein and radial artery harvesting on human cadaveric limb specimens. We used whole leg and whole arm specimens for the teaching in this course because the endoscopic system we have used required carbon dioxide insufflation. They have also had chance to experience different types of harvesting systems including Maquet ${ }^{\circ}$, Sorin ${ }^{\bullet}$ and Terumo ${ }^{\circ}$. The company have provided their internationally experienced trainers to teach in our course. We have used one cadaveric specimen per two participants. Each candidate carried out partial dissection and conduit cauterisation to get experience of the surgical procedure. Every candidate also had chance to practise on the gelatine leg model before the cadaveric specimens.

\section{Median sternotomy and sternal closures}

Full porcine thorax including head and neck was used for median sternotomy. Each participant carried out sternotomy using battery operated sternal saw supplied courtesy of Stryker ${ }^{\circledR}$ Medical plc. (Amsterdam, Netherlands). Each candidate performed skin incision, diathermy dissection and sternotomy and sternal closures using 5 steel Ethicon wires.

\section{Mitral and aortic valve replacement}

The candidates have been taught the full anatomy of the valves, valve resection and valve replacement in the pig heart. Two candidates paired up and one performed surgery and another candidate acted as a first assistant then vice versa. The candidates experienced continuous valve suturing with $2 / 0$ prolene and interrupted valve suturing with $2 / 0$ pledgeted ethibond sutures. Different sizes of mechanical and tissue valves were donated for teaching by Medtronic ${ }^{\bullet}$, plc. (Minneapolis, USA).

\section{Endovascular computerised simulation}

We have used high-fidelity virtual -reality simulators as well as table-top endoscopic models for this session. It consists of various endovascular basic suturing, dissections, cauterisation and vascular anastomosis. The timings and the perfection was automatically calculated by the computer programme and instant feedback was given to the candidates.

\section{Doppler conduit mapping skills}

This session was taught by an experienced vascular scientist from University Hospital of South Manchester NHS Foundation Trust. The candidates were given a brief introduction about the Doppler vascular anatomy of the radial artery and great/lesser saphenous veins. The candidates acted as a real model and the candidates scanned the arms 
and legs of the fellow candidates. In this session, the identification and location of the arteries/vein, depth and length perception of the conduit was taught.

\section{Assessment of procedural knowledge and satisfaction}

The candidates were asked to self-rate their understanding of the surgical procedures on a 5 point Likert scale [4] which included extremely limited understanding and full ability to perform the 12 different surgical procedures. $(1=$ no understanding/could not perform; 2 = extremely limited understanding/ability to perform; $3=$ moderate understanding/ability to perform; 4 = good understanding/ ability to perform; 5 = excellent understanding/ability to perform independently). The pre-course assessments of surgical procedural knowledge were completed online and sent to the organiser a month before the course.

The candidates were also asked to fill in the post-course questionnaire using a Likert scale from 1-5 to evaluate all topics (enrolment procedures, explanation of the course learning objectives, depth of the course content, course booklets, handouts, teaching method used, feedback and tutor interaction of each session and finally resources of the course). In addition, they were also asked to complete a satisfaction questionnaire regarding their learning on a Likert scale of 1-5. Finally, they also reported how confident they were in regard to patient outcomes being improved by attending this type of course.

\section{Statistical analysis}

All data acquired through the questionnaires were analysed using Prism 7.01 software (GraphPad, La Jolla, CA, USA). All descriptive data is expressed as mean \pm standard deviation.

\section{Result}

\section{Pre-course understanding of topics}

The understanding of attendees was assessed for a range of topics and scored from 1-5. Understanding of most topics was relatively low, with cadaveric anatomy scoring the lowest (1.78 \pm 0.96 , Figure 1$)$. Mean scores for the remaining topics are as follows: bronchoscopy $(2.07 \pm$ $1.04)$, chest drain insertion $(1.89 \pm 0.94)$, open thoracotomy $(1.98 \pm$ $0.98)$, VATs wedge resection $(1.93 \pm 1.00)$, laparoscopic skills $(1.87 \pm$ $0.93)$, endoscopic vein harvesting $(1.86 \pm 0.90)$, endoscopic radial harvesting $(1.88 \pm 0.94)$, sternotomy $(1.90 \pm 0.92)$, sternal closure $(1.83$ $\pm 0.98)$, mitral/aortic valve replacement $(1.86 \pm 0.89)$ and vein mapping skills $(1.87 \pm 0.96)$.

\section{Post-course satisfaction with the course}

Satisfaction with the logistics of the course was consistently high, with the following scores: enrolment procedure $(4.62 \pm 0.60)$, explanation of course objectives $(4.59 \pm 0.63)$, depth of course content (4.45 \pm 0.67$)$, teaching methods used $(4.58 \pm 0.64)$, feedback and tutor interaction received $(4.53 \pm 0.66)$ and resources used for the course $(4.55 \pm 0.66$, Figure 2$)$.

\section{Satisfaction with level of learning on the course}

Attendees' satisfaction with the level of learning of each topic was recorded. The highest satisfaction was recorded for the bronchoscopy session $(4.70 \pm 0.52)$. The other topics was scored as follows: chest drain insertion $(4.60 \pm 0.65)$, open thoracotomy $(4.63 \pm 0.60)$, VATs wedge resection ( $4.62 \pm 0.53)$, cadaveric anatomy session (4.63 \pm 0.51$)$, laparoscopic skills $(4.59 \pm 0.62)$, endoscopic vein harvesting $(4.69 \pm$ $0.52)$, endoscopic radial artery harvesting (4.60 \pm 0.65$)$, sternotomy

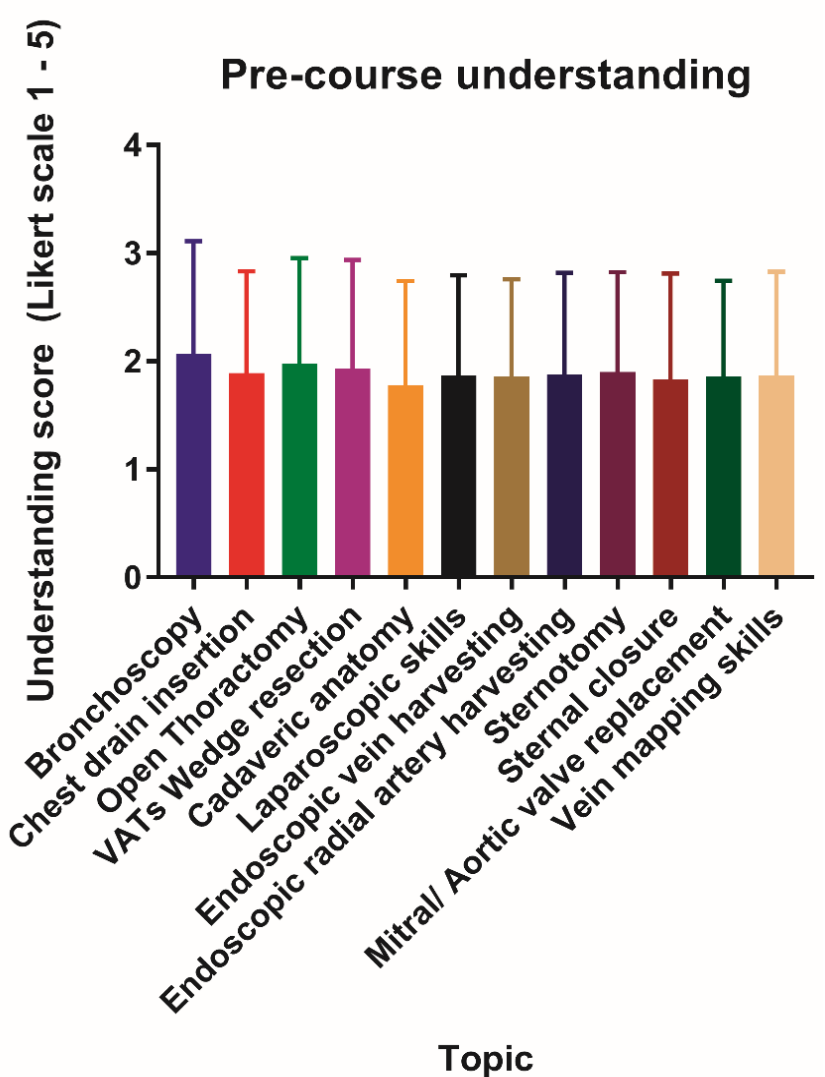

Figure 1. Pre-course understanding of the course topics

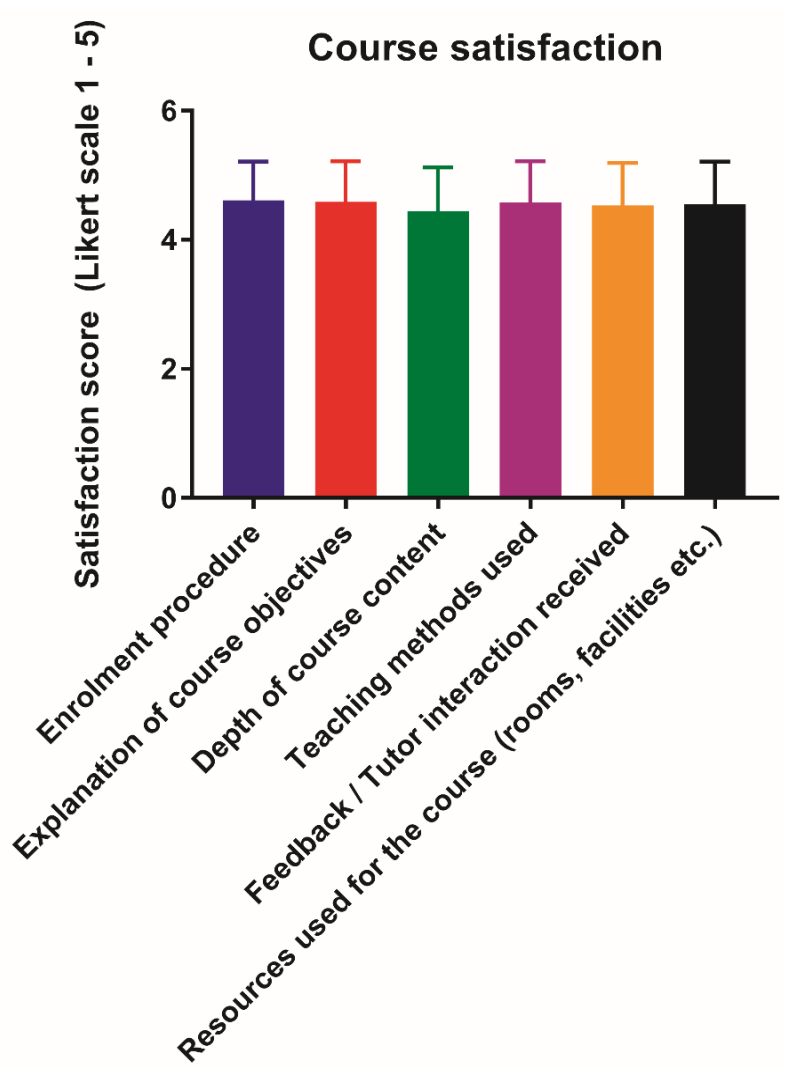

Figure 2. Participants' satisfaction with the course setup and structure 
$(4.60 \pm 0.58)$, sternal closure $(4.62 \pm 0.62)$, mitral/aortic valve replacement $(4.60 \pm 0.62)$ and vein mapping skills (4.63 \pm 0.58 , Figure $3)$.

\section{Confidence in skills and recommendation}

Overall, $92.68 \%$ of attendees were confident that patient outcomes would improve as a result of the course. Additionally, there was a high likelihood of recommending the course to colleagues when scored on a scale of $1-10(9.21 \pm 1.12)$.

\section{Discussion}

For a decade, the benefit of wet lab and simulation-based training has been developing gradually as an integral part of cardiothoracic surgery training. To achieve full clinical competency, the trainees need to learn surgery on real patients, which puts huge pressure on patient safety if the trainee does not fully understand the complex knowledge of the surgical procedures [5]. The number of adverse events has also been decreased after trainees get hands-on practice in a stress free environment [6].

The pre-course survey suggests that there is a real need for cardiothoracic procedural knowledge and competency training to improve their surgical skills. However, there are a lot of barriers to setting up these courses which involves a considerable amount of dedicated time from the senior consultants and high cost. A systematic review highlights that the main obstacles to implementing these trainee programmes are lack of infrastructure, faculty time and cost [7]. From our experience, it is clear that proper planning, involving the full educational surgical team and relevant surgical companies can be very beneficial and these courses can be run successfully to achieve the training goal.

This is the first intensive CT course survey report exploring the feedback results of 15 consecutive courses. Our post course findings

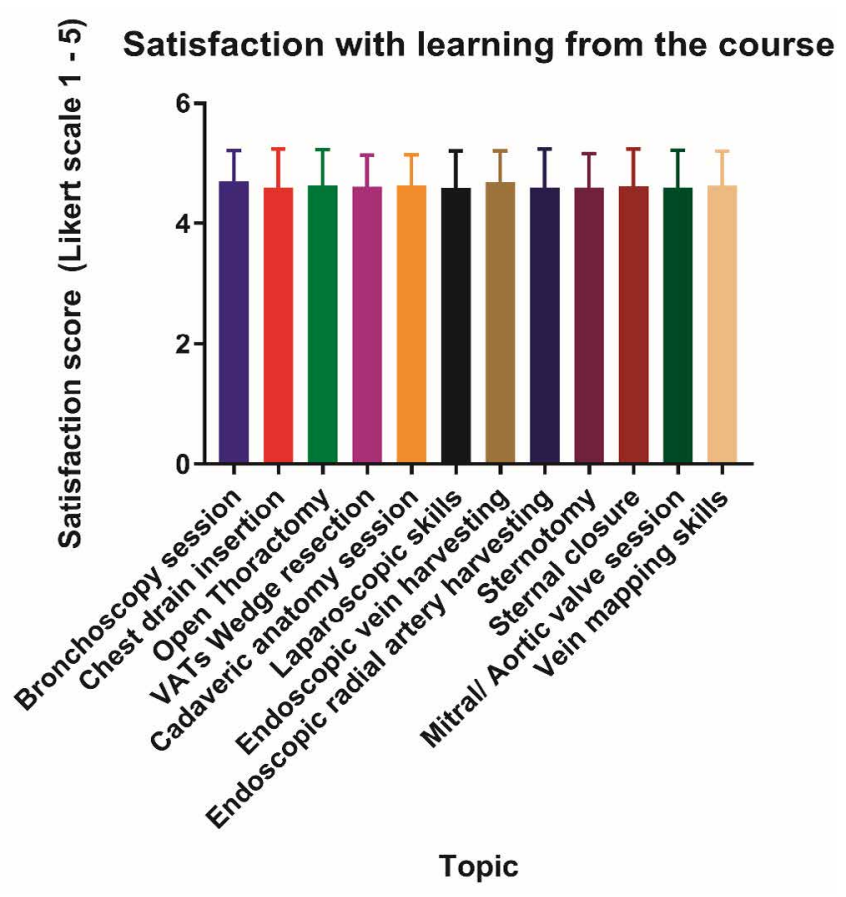

Figure 3. Participants' satisfaction with the level of learning achieved from the course suggest that course satisfaction was consistently high across the board with participants rating the course setup and logistics highly. In addition, all participants were satisfied with the level of learning achieved through the course and were all confident that they would recommend the course to their colleagues.

The level of trainee and practitioners' previous experiences did not affect the course feedback because the course curriculum was set for certain core trainees and senior practitioners. We had strict entry criteria to avoid very senior core trainees who are at the last level of their training period and trainee surgical care practitioners who had not been exposed to CT surgery. Studies have highlighted that intensive simulation courses can benefit both junior and senior trainees $[4,8]$ but from our previous experiences, more senior trainees dilute or overtake the junior trainees and practitioners. This puts pressure on and increases stress during the training period. We have learned this from our previous delegates' course feedback. So, this study was limited to CT1, CT2, ST1, ST2 and surgical care practitioners' level.

Our delegates have also had an opportunity to benchmark and share their experiences with each other during their hands-on practices. The structure of the course is crucial for obtaining full benefit of the programmes. Our courses were set according to their trainees' national core curriculum. We have also designed the course to cover elements from basic anatomy to high fidelity hands-on computer skills.

The limitation of these courses was that the post course knowledge evaluation was not performed. We have also not recorded the outcome of tests performed during the course, which would have been useful for evaluation. Our aim of this paper is to highlight the importance of these courses in cardiothoracic surgery and promote the benefits of investing in such courses. We also believe that the long-term effects of these courses should be evaluated by following these trainees to their workplace and getting feedback over a longer timescale. We have not explored this in these courses due to the cost and time involved. However, this area needs to be investigated in future.

In conclusion, our intensive curriculum aligned CT courses had more hands-on wet lab sessions than didactic lectures. Our delegates felt that these types of courses will increase the chances of improved patient outcomes. It is important to teach the trainees with wet lab hands-on courses outside the theatre settings regularly.

\section{Conflicts of interest}

None.

\section{Source of funding}

There was no fund given by any funders for this study. However, all the fifteen surgical courses were funded by Society of Cardiothoracic Surgeons (all faculties, administration support and advertisements), Ethicon International (all course fees, travel, accommodation for the students and faculties, Animal cadaveric tissues, venue, food and beverages), Maquet International (human cadaveric tissues), Terumo International(human cadaveric tissues), Stryker (sternal saw, batteries and saw blades), Karl Storz (TV, stack monitoring systems, bronchoscopy equipments, camera and computer surgical skills stations), Medtronic (pig's heart, mechanical/biological valves and valve instruments) and Rocket Medicals (chest drains and chest drain systems). 


\section{References}

1. Reznick RK, MacRae H (2006) Teaching surgical skills-changes in the wind. $N$ Engl J Med 355: 2664-2669. [Crossref]

2. Reznick RK (1993) Teaching and testing technical skills. Am J Surg 165: 358-361. [Crossref]

3. Ericsson KA (2004) Deliberate practice and the acquisition and maintenance of expert performance in medicine and related domains. Acad Med 79: S70-81. [Crossref]

4. Robinson WP, Doucet DR, Simons JP, Wyman A, Aiello FA, et al. (2017) An intensive vascular surgical skills and simulation course for vascular trainees improves procedural knowledge and self-rated procedural competence. J Vasc Surg 65: 907-915. [Crossref]
5. Singh H, Thomas EJ, Petersen LA, Studdert DM (2007) Medical errors involving trainees: a study of closed malpractice claims from 5 insurers. Arch Intern Med 167: 2030-2036. [Crossref]

6. Morgan PJ, Cleave-Hogg D (2002) A worldwide survey of the use of simulation in anesthesia. Can J Anaesth 49: 659-662. [Crossref]

7. Mitchell EL, Arora S, Moneta GL, Kret MR, Dargon PT, et al. (2014) A systematic review of assessment of skill acquisition and operative competency in vascular surgical training. J Vasc Surg 59: 1440-1455. [Crossref]

8. Pandey VA, Black SA, Lazaris AM, Allenberg JR, Eckstein HH, et al. (2005) Do workshops improved the technical skill of vascular surgical trainees? Eur $J$ Vasc Endovasc Surg 30: 441-447. [Crossref]

Copyright: (C2019 Krishnamoorthy B. This is an open-access article distributed under the terms of the Creative Commons Attribution License, which permits unrestricted use, distribution, and reproduction in any medium, provided the original author and source are credited. 